\title{
Editorial
}

\section{Acknowledgement to Reviewers of Antioxidants in 2014}

Antioxidants Editorial Office, MDPI AG, Klybeckstrasse 64, CH-4057 Basel, Switzerland

Published: 7 January 2015

The editors of Antioxidants would like to express their sincere gratitude to the following reviewers for assessing manuscripts in 2014:

\begin{tabular}{|c|c|c|}
\hline Abourashed, Ehab & Bullon, Pedro & Donsì, Francesco \\
\hline Adjé, Félix & Cabrera, Carmen & Dunn, Robert O. \\
\hline Aggelis, George & Camacho, M. en C. Antonio & Durazzo, Alessandra \\
\hline Ahmed, Faiyaz & Nieto & Esposto, Sonia \\
\hline Aksakal, Ercüment & Cao, Wei & Estévez, Mario \\
\hline Alvarez-Suarez, José Miguel & Carrì, Maria Teresa & Fassett, Robert \\
\hline Amarowicz, Ryszard & Caruso, Francesco & Fleenor, Bradley S. \\
\hline Andrés, A.i. & Castro-Puyana, María & Franck, Thierry J. \\
\hline Annibalini, Giosuè & Cateni, Francesca & Franco, Rodrigo \\
\hline Ashida, Hitoshi & Cavar, Sanja & Frank, Jan \\
\hline Azimzadeh, Omid & Céspedes, Carlos L. & Fraser, Paul \\
\hline Bahorun, Theeshan & Chemat, Farid & Fregapane, Giuseppe \\
\hline Barreiro, Sonia Losada & Cheng, Jen-Hua & Gan, Chee-Yuen \\
\hline Barreto, Maria Do Carmo & Choy, Ying & Gatz, Christiane \\
\hline Battino, Maurizio & Chyau, Charng-Cherng & Gibson, Claire L. \\
\hline Beaulieu, Michaël & Coelho, José A & Gibson, Deanna \\
\hline Belin, Christophe & Combet, Emilie & Gordon, Michael \\
\hline Beretta, Giangiacomo & Cortopassi, Gino A. & Gottschalk, Leda Maria Fortes \\
\hline Bernini, Roberta & Costabile, Adele & Grilli, Alfredo \\
\hline Bhat, Rajeev & Cozzolino, Mauro & Gupta, Vivek \\
\hline Birch, Edward & Cuman, Roberto Kenji & Hamdy Roby, Mohamed \\
\hline Böhm, Volker & Nakamura & Hussein \\
\hline Borras, Consuelo & Custódio, Luísa & Hasanuzzaman, Mirza \\
\hline Bradshaw, Patrick C. & Day, Billy & Hasegawa, Noboru \\
\hline Bravo-Díaz, Carlos & De La Cruz, José Pedro & Hauer-Jensen, Martin \\
\hline
\end{tabular}


Hemalatha, Siva

Hernandez, Tamara Yulieta

Forbes

Holuigue, Loreto

Huang, Yu

Hüttemann, Maik

Iwasaki, Masanori

Jayasinghe, Chamila

Jirillo, Emilio

Jongberg, Sisse

Jordán, María José

Juranić, Zorica D

K Pandey, Abhay

Khowala, Suman

Kita, Agnieszka

Kocsy, Gabor

Koeppen, Arnulf

Komarov, Andrei M

Kozlowski, Henryk

Kremer, Dario

Kumar, Anil

Lachman, Jaromir

Lage, Miguel Ángel Prieto

Lalas, Stavros

Landi, Marco

Lash, Larry

Legarreta, I. Guerrero

Leite, Edda Lisboa

Li, Jian Jian

López-Jornet, Pía

Lorenzi, V

Lores Aguin, Marta

Makris, Dimitris P.

Mao, Qingcheng

Martin, Pamela M.

Mascitelli, L.

Mateos, Raquel

Matés, José M.

Matsuguchi, Tetsuya

Maurizi, Charles P.

Mazzoni, I.

Méndez-álvarez, Estefanía
Merah, Othmane

Miller, Alyson

Miller, David S.

Mirunalini, Sankaran

Mohamed Jalil, Abbe Maleyki

Mohamed, RAMDANI

Morales, Juan C.

Naito, Yuji

Nerín, Cristina

Neufeld, Howard S.

Niewold, Theo

Nino, Russo

Niwa, Toshio

Obana, Akira

Oda, Tatsuya

Odriozola-Serrano, Isabel

Olcese, James

Opara, Elizabeth I.

Osakabe, Naomi

Panfoli, Isabella

Panzella, Lucia

Paraskeva, Christakis A.

Park, Miyoung

Peiró, Sara

Pereira, Eliana

Pereira, Vanda

Pizza, Cosimo

Poerschmann, Juergen

Polikarpov, Igor

Potenza, Lucia

Prasad, Majeti Narasimha Vara

Priefer, Ronny

Proestos, Charalampos

Rai Vittal, Ravishankar

Rai, Dilip K.

Ramadan Hassanien,

Mohamed Fawzy

Ramos, Sonia

Ravi, Sajja

Reddy, P. Hemachandra

Remelli, Maurizio

Rodrigues, Francisca
Rodrigues, Gerard A.

Romero, Francisco Javier

Roseiro, Luísa B.

ROUANET, Jean-Max

Routray, Winny

Sahin, Selin

Salvador, Mirian

Sarker, Satya

Schauss, Alexander G.

Schempp, Harald

Serralheiro, Maria Luísa M.

Servili, Maurizio

Shang, Fu

Shen, Chwan-Li

shen, xiangchun

Shin, Sung Chul

Shukla, Shruti

Siger, Aleksander

Simal-Gándara, Jesús

Singh, Suneel Kumar

Sola, Rosa

Sørensen, Ann-Dorit Moltke

Sousa, Carla

Spitz, Douglas

Srivatsan, Malathi

Srzednicki, George

Stagkos, Dimitri

Stamatopoulos, Konstantinos

Summo, Carmine

Szymula, Marta

Taamalli, Ameni

Thiyam, Usha

Tundis, Rosa

Ufer, Christoph

Van Duynhoven, John P. M.

VanHorssen, Jack

Varzakas, Theodoros

Verardo, Vito

Villeneuve, Pierre

Wang, Dayong

Weber, John T

Wilhelm, Ethel 

$\mathrm{Wu}$, Chi-Rei
Yamaguchi, Fumio
Zhang, Longze
$\mathrm{Wu}, \mathrm{Li}-\mathrm{Chen}$
Zervoudakis, George

(C) 2015 by the authors; licensee MDPI, Basel, Switzerland. This article is an open access article distributed under the terms and conditions of the Creative Commons Attribution license (http://creativecommons.org/licenses/by/4.0/). 